subsequently retrieved via cut down method in an emergency operation.

Results A 28-week-gestational age male infant presented with intestinal perforation requiring emergency laparotomy and ileostomy formation. At 3 months of age (corrected age 40 weeks), he required intravenous nutrition due to high output stoma and suboptimal weight gain. Insertion of a vygon Premicath 1 French catheter via the left cephalic vein at the forearm was attempted. X ray showed complex coiling of the catheter within the vein (figure 1), therefore it was decided to remove the catheter at the bedside, however, resistance was encountered when withdrawing the catheter. Xray confirmed knotting of the catheter at about $6 \mathrm{~cm}$ away from the percutaneous puncture site (figure 2).

Emergency operation under general anesthesia was arranged, and venotomy was made directly over the knot after controlling the left cephalic vein. The catheter was retrieved and the cephalic vein was ligated afterwards. Post op recovery was uneventful. Subsequently a tunneled central catheter (Broviac catheter) was inserted at an elective operation into the right internal jugular vein

Conclusions PICC have been used extensively in children where vascular access can often be challenging for medium term infusion therapy. These are non-tunneled vascular devices that can be inserted at the bedside (1). Knot formation during PICC insertion in a 5-month old infant had been reported (2) and the author suggested a trial of knot dissolution by repeated flushing with saline solution. Another paper suggested using 0.008' hydrophilic guidewire to unloop the PICC knot, however it was not actually performed as the case described did not have any real knot (3). Our case illustrated the potential complication of PICC insertion in a small neonate.

\section{MORTALITY TRENDS OF ONCOLOGY \& HEMATOPOIETIC STEM CELL TRANSPLANT PATIENTS SUPPORTED ON EXTRACORPOREAL MEMBRANE OXYGENATION: A SYSTEMATIC REVIEW \& META-ANALYSIS}

RR Pravin, Benjamin Xiongzheng Huang. Singapore

\subsection{6/bmjpo-2021-RCPCH.172}

Background There is an increasing frequency of oncology and hematopoietic stem cell transplant (HSCT) patients seen in the intensive care unit and requiring extracorporeal membrane oxygenation (ECMO), however, prognosis of this population over time is unclear. This study aims to determine the mortality trends and complication rate in oncology and HSCT patients receiving ECMO by conducting a systematic review and meta-analysis.

Objectives The main outcome was all-cause mortality and studies reporting mortality within any timeframe (e.g. survival to decannulation, ICU, hospital, 28-, 60- and 90-day mortality, etc.) were included. However, hospital mortality was the most clinically relevant and was used as the primary outcome in the analyses. Secondary outcomes included bleeding, mechanical, cardiovascular, pulmonary, neurological and renal complications on ECMO, defined by the ELSO registry database definitions, and duration of ECMO, mechanical ventilation (MV) and ICU stay.

Methods MEDLINE, EMBASE, Cochrane and Web of Science were searched from earliest publication until April 10, 2020 for studies to determine the mortality trend over time in oncology and HSCT patients requiring ECMO. Primary outcome was hospital mortality. Random-effects meta-analysis model was used to obtain pooled estimates of mortality and 95\% confidence intervals. A priori subgroup metanalysis compared adult versus pediatric, oncology versus HSCT, hematological malignancy versus solid tumor, allogeneic versus autologous HSCT, and veno-arterial versus veno-venous ECMO populations. Multivariable meta-regression was also performed for hospital mortality to account for year of study and HSCT population.

Results 17 eligible observational studies ( $n=1109$ patients) were included. Overall pooled hospital mortality was 72\% (95\% CI: 65, 78). In the subgroup analysis, only HSCT was associated with a higher hospital mortality compared to oncology subgroup [84\% (95\% CI: 70, 93) vs. 66\% (95\% CI: 56, 74); $p=0 \cdot 021]$. Meta-regression showed that HSCT was associated with increased mortality [adjusted odds ratio (aOR) 3.84 $(95 \%$ CI $1.77,8.31)]$ but a later year of study was associated with decreased mortality [adjusted odds ratio (aOR) 0.92 (95\% CI: 0.85, 0.99)].

Conclusions This study reports a high overall hospital mortality in oncology and HSCT patients on ECMO which improved over time. The presence of HSCT portends almost a fourfold increased risk of mortality and this finding may need to be taken into consideration during patient selection for ECMO.

\section{DISPARITY BETWEEN DIFFERENT REFERENCE VALUES FOR HYPERTENSION AMONG CHILDREN AND ADOLESCENTS TREATED FOR ADHD: EXPERIENCE FROM A NORTH WEST ENGLAND CCH CLINIC}

Michael Ogundele, Cornelius Ani. UK

\subsection{6/bmjpo-2021-RCPCH.173}

Background ADHD affects 5\% of children and young people (CYP) and, if untreated, can be associated with significant multi-domain impairments. Medications licensed for ADHD treatment are effective but can be associated with side effects. Stimulant medications (Methylphenidate and Dexamfetamine/Lisdexamfetamine) and Atomoxetine are associated with small but statistically significant increases in Blood Pressure (BP) and Pulse. Intuniv is an alpha 2a agonist, which can be associated with low BP and bradycardia. Thus, guidelines recommend regular monitoring of BP and Pulse and plotting the figures on reference centile charts. However, confusion can arise about which reference centiles to use due to differences in the cut-offs applied in different regions of the world. Both the American Academy of Pediatrics (AAP) and European Society of Hypertension (ESH) define hypertension (HT) in CYP as BP $\geq 95$ th percentile for gender, age and height on three or more separate occasions, while pre-hypertension (PreHT) (now redefined as elevated $\mathrm{BP}$ ) is $\mathrm{BP} \geq 90$ th percentile but less 95 th percentile. However, the British reference for CYP between 4 and 23 years defined HT as BP above the 98th percentile for age, and 'high-normal BP' (PreHT) between the 91st and 98th percentile.

Objectives This audit aimed to describe the BP profile of a sample of otherwise healthy CYP on medications for ADHD attending routine clinical reviews, and to compare the 
proportions meeting cut-off for HT or PreHT/'Elevated BP' (EBP) based on British vs AAP/ESH reference definitions.

Methods Between March 2019 and March 2020, 67 CYP who attended routine clinician-led appointments for ADHD medication review had their BP and Pulse measured with electronic sphygmomanometers. The equipment was regularly calibrated and clinicians followed standard procedures for checking BP and Pulse.

Results Of the 67 CYP, 85\% were males, and the average age of the cohort was 12 years. Based on the British definition, $12(18 \%)$ were recorded as PreHT while $7 \%$ met the criteria for HT. The corresponding proportion of CYP meeting the AAP/ESH definitions for EBP and HT were 15\% and 7.5\% respectively. the CYP with HT or PreHT were monitored with non-clinic based BP measurements at home or by the GP and all returned to normal in the following eight months.

Conclusions The proportions of CYP classified by the British or AAP/ESH reference as having HT or PreHT were different albeit with small margins. However these small differences in proportion could have population-level implications if mapped onto the full cohort of CYP with ADHD attending similar medication reviews in the UK. Also, the differences could lead to frontline clinicians using different thresholds for triggering remedial actions in CYP with suspected HT such as medication dose reduction or paediatric cardiology referral. Therefore, we recommend further review of these different reference points to avoid confusion among frontline clinicians. One potential reason for the differences is that the British reference does not consider the CYP's height. Perhaps, including height in the algorithm that determines all BP reference centiles for CYP would provide closer results.

\section{LONGITUDINAL STUDY OF BLOOD PRESSURE AMONG CHILDREN AND ADOLESCENTS WITH ADHD SUGGESTS HIGH LEVELS OF STRESS AND ANXIETY DURING THE COVID-19 PANDEMIC LOCKDOWN}

Michael Ogundele. UK

\subsection{6/bmipo-2021-RCPCH.174}

Background The whole world is experiencing an unprecedented period of severe social disruption and economic downturn due to global spread of a rare new strain of Severe Acute Respiratory Syndrome (SARS) virus called Coronavirus. Children and Young people (CYP) are less likely to have severe symptoms from acute COVID-19 infections, but they are more prone to possible several adverse mental health outcomes. Social isolation (eg, marital status, number of close friends and relatives, religious or other group affiliations) is known to be a major cause of stress and risk factor for cardiovascular disease including elevated Blood Pressure (BP) and Heart rates (HR).

Objectives This study aimed to analyse the effect of the UK pandemic national lockdown on the cardiovascular health (CVH) of CYP with ADHD regularly followed up in a North West England Community Child Health Clinic. We compared the BP and HR measurements for the CYP before and after the COVID-19 pandemic lockdown (CPL) to assess its effects on their $\mathrm{CVH}$.
Methods The CVH of 57 CYP with ADHD was prospectively studied during their clinic reviews, before and after the official commencement of the national UK lockdown on the 26th March 2020. Pre-Hypertension (PreHT)/Elevate BP (EBP) and Hypertension (HT) were defined using both the Great Britain reference charts (between 91st and 98th centile or above 98th centile for age) and the US-based recommendations (between 90th and 95th centile or above 95 percentile for gender, age and height) respectively. The centiles for HR measurements were derived from the published reference data from existing international studies of normal children. Student paired T-test was used for comparison of pre/post CPL BP/HR data.

Results The clinical characteristics of CYP seen before and after the CPL were similar in terms of average age, number of co-morbidities, use of medications and gender distribution.

The HR, diastolic and systolic BP of all the CYP with ADHD significantly increased during the CPL $(p<0.001)$. There was two- to four-fold increase in the number of the CYP with HT, PreHT/EBP measured after the CPL (figure 1). Diastolic BP elevation accounted for $89 \%$ to $100 \%$ of the abnormal BP measurements. This increase in measured $\mathrm{BP}$ and HR could not be attributed to any prescribed ADHD medications as the patients had been on the same medications and unchanged dosage for an average of more than 16 months, all within the lower range of normal doses.

There was statistically significant correlation between the elevated systolic and diastolic BP, and the number of weeks from the inception of the CPL.

All the CYP with elevated BP are being closely monitored in the clinic and at home. The elevated BP measurements have gradually resolved as the lockdown was eased and no long-term negative consequences have been observed.

Conclusions We conclude that the CPL was associated with transient and reversible increase in the BP and HR of CYP with background diagnosis of ADHD and other co-morbid neurodevelopmental disorders. This is probably an indication of the psychological distress associated with the pandemic affecting CYP, as also previously described for adults.

\section{BEFORE SCALING-UP, SPEAK WITH THE STAKEHOLDERS: LESSONS FROM A PRETERM PROJECT}

Josephine Agyeman-Duah, Anita Appiah, Jacqueline Asibey, Ernest Asiedu, Stephen Kennedy, Jose Villar. UK

\subsection{6/bmjpo-2021-RCPCH.175}

Background In 2019, the [1]National Catholic Health Service (NCHS) initiated the INTERPRACTICE-21st Study in Ghana: implementation of the [2]INTERGROWTH-21st (IG21) Preterm Postnatal Growth Standards and Feeding Protocols within its hospitals.

Implementation included

1. Monitoring the growth of preterm babies with the IG21 standards, rather than the traditional growth charts for term babies.

2. Training health professionals involved with neonatal care to use the IG21 standards and evidence-based, feeding protocols (with their focus on the use of breast milk). 